\title{
VORWORT ZUR RUSSISCHEN AUFLAGE
}

Der vorliegende abschließende Band der ,Theoretischen Physik“ befaßt sich mit der physikalischen Kinetik, die im weiten Sinne als mikroskopische Theorie der Prozesse in statistischen Nichtgleichgewichtssystemen verstanden wird.

Im Unterschied zu den Eigenschaften statistischer Gleichgewichtssysteme sind die kinetischen Eigenschaften wesentlich enger mit dem Charakter der mikroskopischen Wechselwirkungen der speziellen physikalischen Objekte verknüpft. Daher rührt die gewaltige Mannigfaltigkeit dieser Eigenschaften und der wesentlich höhere Schwierigkeitsgrad ihrer theoretischen Deutung. Aus diesem Grunde läßt sich auch die Frage nach der Auswahl des Stoffes, welcher in einem allgemeinen Lehrgang der Theoretischen Physik enthalten sein soll, weniger eindeutig beantwortén.

Der Inhalt des Buches ist aus dem Inhaltsverzeichnis ersichtlich. Wir wollen in diesem Zusammenhang nur einige Bemerkungen anfügen.

Besondere Aufmerksamkeit wird in diesem Buch der Theorie der Gase als dem vom Prinzip her einfachsten Objekt der kinetischen Theorie gewidmet. Einige Kapitel beschäftigen sich mit der Plasmatheorie - nicht allein im Hinblick auf die physikalische Bedeutung dieses Teiles der Kinetik, sondern auch deshalb, weil viele Aufgaben der Plasmakinetik bis zum Ende gelöst werden können und eine lehrreiche Illustration der allgemeinen Methoden der kinetischen Theorie bieten.

Besonders vielfältig sind die kinetischen Eigenschaften in Festkörpern. Wir mußten uns natürlich bei der Auswahl des Stoffes für die entsprechenden Kapitel auf die allgemeinsten Fragen beschränken, welche die grundlegenden kinetischen Vorgänge und Methoden ihrer Behandlung aufzeigen. Wir möchten in diesem Zusammenhang nochmals unterstreichen, daß dieses Buch Teil eines Lehrgangs für Theoretische Physik ist und nicht den Anspruch erhebt, ein Lehrwerk für Festkörpertheorie zu sein.

Im Inhalt dieses Buches gibt es zwei offensichtliche Lücken. Es fehlen die Fragen der Kinetik magnetischer Prozesse und die Theorie der kinetischen Erscheinungen, die mit dem Durchgang schneller Teilchen durch Materie verbunden sind. Diese Lücken sind durch den Mangel an Zeit bedingt, und wir haben uns entschieden, sie in dieser Ausgabe zu belassen, damit die Herausgabe des Buches nicht noch mehr hinausgezögert wird. Wir möchten der Hoffnung Ausdruck geben, daß, obgleich auf diese Weise in dem Buch nicht alles, was erforderlich wäre, enthalten ist, all das, was in ihm enthalten ist, von Interesse und für die Leser von Nutzen sein wird.

Dieses Buch bringt ein Programm zum Abschluß, welches von Lew DAwIDowitsch LANDAU vor mehr als vierzig Jahren entworfen wurde. Der gesamte Lehrgang besteht aus folgenden Bänden: 
Band I. Mechanik

Band II. Klassische Feldtheorie

Band III. Quantenmechanik (nichtrelativistische Theorie)

Band IV. Relativistische Quantentheorie

Band V. Statistische Physik, Teil 1

Band VI. Hydrodynamik

Band VII. Elastizitätstheorie

Band VIII. Elektrodynamik der Kontinua

Band IX. Statistische Physik, Teil 2

Band X. Physikalische Kinetik

Es soll darauf hingewiesen werden, daß die Anordnung von Band IX in dieser Reihe dadurch bedingt ist, daß in ihm in beträchtlichem Umfang Kenntnisse aus der Hydrodynamik und der makroskopischen Elektrodynamik benutzt werden.

In der neuen Auflagenreihe, die 1973 begonnen wurde ${ }^{1}$ ), sind bisher die Bände I, II, III, V, IX und $\mathrm{X}$ erschienen. Band VII kann ohne große Veränderungen wiederaufgelegt werden. Aus dem Band IV, der bisher unter dem Titel „Relativistische Quantentheorie" herausgegeben wurde, werden die Abschnitte zur schwachen und zur starken Wechselwirkung herausgenommen, und dieser Band wird demnächst als „Quantenelektrodynamik“ wiederaufgelegt. Die Bände VI und VIII, die schon im Verlaufe vieler Jahre nicht wiederaufgelegt wurden, erfordern dagegen eine umfangreichere Utberarbeitung und Ergänzung. Wir beabsichtigen, uns mit diesen Dingen in der nächsten Zeit zu befassen.

Unser herzlicher Dank gilt A. F. Andrejew, R. N. Gurshr, W. L. GuRewitsch, J. M. KagAN, M. I. Kaganow und I. M. Lrfschitz, mit denen wir die in diesem Buch behandelten Fragen erörtert haben.

L. P. Gorkow und A. A. Ruchadse, die das Buch im Manuskript durchgesehen und einige Bemerkungen gemacht haben, sind wir zu Dank verpflichtet.

November 1978

E. M. LifSCHITZ

L. P. Pitajewski

\section{ZUR DEUTSCHEN AUSGABE}

Die deutsche Utbersetzung dieses Bandes wurde von Dr. G. Drener redigiert. Wir möchten ihm unseren aufrichtigen Dank für seine Sorgfalt und die im Laufe der Arbeit gemachten wertvollen Bemerkungen aussprechen.

September 1981

E. M. Lifschitz

L. P. Pitajewski

1) In deutscher Sprache erscheint die neue Reihe seit 1976 (Anm. d. Hrsg.). 
